\title{
THE NATURAL RIGHT AND THE NATURAL LEFT INVERSES OF RECTANGULAR MATRICES
}

\author{
ALEXANDER ABIAN and PAULA A. KEMP
}

\begin{abstract}
A.bstract. If an $m$ by $n$ with $m<n$ matrix $A$ has a right inverse then it has infinitely many right inverses. In fact, $K(A K)^{-1}$ is a right inverse of $A$ for many $n$ by $m$ matrices $K$ of rank $m$. The natural choice for $K$ is the transpose $A^{\prime}$ of A. Thus, we call $A^{\prime}\left(A A^{\prime}\right)^{-1}$ the natural right inverse of $A$. It can be used (not so obviously) to solve $A X=C$ yielding the solution $X=A^{\prime}\left(A A^{\prime}\right)^{-1} C$ which minimizes the length $\|X\|$. Similarly, if an $n$ by $m$ with $m<n$ matrix $B$ has a left inverse, we call $\left(B^{\prime} B\right)^{-1} B^{\prime}$ the natural left inverse of $B$. It can be used (in an obvious way) in an attempt "to solve" $B X=C$ yielding the best approximate solution $X=\left(B^{\prime} B\right)^{-1} B^{\prime} C$ which minimizes the error $\|C-B X\|$.
\end{abstract}

In what follows, the entries of all matrices are real numbers.

Let us consider a 2 by 3 matrix $A$, say, $\left(\begin{array}{lll}0 & 1 & 0 \\ 1 & 0 & 2\end{array}\right)$. It is customary to call a 3 by 2 matrix $R$ a right inverse of $A$ if $A R=I_{2}$ where $I_{2}$ is the 2 by 2 unit matrix. For instance, since

$$
\left(\begin{array}{lll}
0 & 1 & 0 \\
1 & 0 & 2
\end{array}\right)\left(\begin{array}{cc}
-2 & -3 \\
1 & 0 \\
1 & 2
\end{array}\right)=\left(\begin{array}{ll}
1 & 0 \\
0 & 1
\end{array}\right) \text { and }\left(\begin{array}{lll}
0 & 1 & 0 \\
1 & 0 & 2
\end{array}\right)\left(\begin{array}{cc}
2 & -5 \\
1 & 0 \\
-1 & 3
\end{array}\right)=\left(\begin{array}{ll}
1 & 0 \\
0 & 1
\end{array}\right)
$$

we set that $\left(\begin{array}{cc}-2 & -3 \\ 1 & 0 \\ 1 & 2\end{array}\right)$ as well as $\left(\begin{array}{cc}2 & -5 \\ 1 & 0 \\ -1 & 3\end{array}\right)$ is a right inverse of $\left(\begin{array}{lll}0 & 1 & 0 \\ 1 & 0 & 2\end{array}\right)$.

It is easy to observe that $\left(\begin{array}{lll}0 & 1 & 0 \\ 1 & 0 & 2\end{array}\right)$ has infinitely many right inverses.

A method of constructing right inverses of the above 2 by 3 matrix $A$ is as follows. First, we multiply $A$ on the right by a suitable 3 by 2 matrix $K$ of rank 2 obtaining the 2 by 2 square matrix $A K$ of rank 2. Clearly, the usual inverse $(A K)^{-1}$ of $A K$ exists. Then, we consider the 3 by 2 matrix $K(A K)^{-1}$ and we claim that it is a right inverse of $A$. But this is obvious since $A K(A K)^{-1}=I_{2}$.

Received August 3, 1987.

1980 Mathematics Subject Classification. Primary 15A06; Secondary 15A09. 
Let us observe that our above construction of a right inverse of the matrix $A=$ $\left(\begin{array}{lll}0 & 1 & 0 \\ 1 & 0 & 2\end{array}\right)$ hinges on the fact that: $A K$ is a sequare matrix and $(A K)^{-1}$ exists

Since the rank of our 2 by 3 matrix $A$ is 2 , we see that (1) is satisfied by infinitely many distinct 3 by 2 matrices $K$ of rank 2 . Thus, indeed our matrix $A$ has infinitely many right inverses. A natural choice for $K$ is the transpose $A^{\prime}$ of $A$. Clearly, $A^{\prime}$ is a 3 by 2 matrix of rank 2 and $A^{\prime}\left(A A^{\prime}\right)^{-1}$ is a right inverse of $A$. However, since $A^{\prime}\left(A A^{\prime}\right)^{-1}$ is uniquely determined by $A$ we call it the natural right inverse of $A$. Accordingly,

$$
\begin{aligned}
& \text { the natural right inverse of }\left(\begin{array}{lll}
0 & 1 & 0 \\
1 & 0 & 2
\end{array}\right) \text { is } \\
& \left(\begin{array}{ll}
0 & 1 \\
1 & 0 \\
0 & 2
\end{array}\right)\left(\left(\begin{array}{lll}
0 & 1 & 0 \\
1 & 0 & 2
\end{array}\right)\left(\begin{array}{ll}
0 & 1 \\
1 & 0 \\
0 & 2
\end{array}\right)\right)^{-1}=\left(\begin{array}{cc}
0 & 1 / 5 \\
1 & 0 \\
0 & 2 / 5
\end{array}\right)
\end{aligned}
$$

Remark 1. It is well known that an $m$ by $n$ (with $m<n$ ) matrix $A$ of rank $m$ is such that $\left(A A^{\prime}\right)^{-1}$ always exists. However, since $m<n$ we cannot replace $\left(A A^{\prime}\right)^{-1}$ by $\left(A^{\prime}\right)^{-1} A^{-1}$ because $A$ is not a square matrix to begin with. On the other hand, if $m=n$, i.e., if $A$ is a square $m$ by $m$ matrix of rank $m$ then $\left(A A^{\prime}\right)^{-1}=\left(A^{\prime}\right)^{-1} A^{-1}$ and consequently the natural right inverse $A^{\prime}\left(A A^{\prime}\right)^{-1}$ of $A$ reduces to the usual inverse $A^{-1}$ of $A$.

Based on the above Remark, we introduce:

Definition 1. Let $A$ be an $m$ by $n$ with $m<n$ matrix of rank $m$. Then the $n$ by $m$ matrix $A^{\prime}\left(A A^{\prime}\right)^{-1}$ where $A^{\prime}$ is the transpose of $A$, is called the natural right inverse of $A$.

Now, we show how the natural right inverse (and for that matter any right inverse) of a matrix can be used to solve a system of $m$ linear equations in $n$ unknowns where $m<n$ and where the rank of the $m$ by $n$ matrix of coefficients of the unknowns is $m$. For instance, let us consider:

$$
\left(\begin{array}{lll}
0 & 1 & 0 \\
1 & 0 & 2
\end{array}\right)\left(\begin{array}{l}
x \\
y \\
z
\end{array}\right)=\left(\begin{array}{c}
5 \\
10
\end{array}\right)
$$

As (2) shows, the natural right inverse of the matrix of coefficients of the unknowns of the system (3) exists and is equal to $\left(\begin{array}{cc}0 & 1 / 5 \\ 1 & 0 \\ 0 & 2 / 5\end{array}\right)$. The question is how to use it to determine $\left(\begin{array}{l}x \\ y \\ z\end{array}\right)$ 
Obviously, multiplication on the right of both sides of the equality sign in (3) by $\left(\begin{array}{cc}0 & 1 / 5 \\ 1 & 0 \\ 0 & 2 / 5\end{array}\right)$ does not yield $\left(\begin{array}{l}x \\ y \\ z\end{array}\right)$. Similary, at first glance, multiplication on the left of both sides of the equality sign in (3) by $\left(\begin{array}{cc}0 & 1 / 5 \\ 1 & 0 \\ 0 & 2 / 5\end{array}\right)$ does not seem to yield $\left(\begin{array}{l}x \\ y \\ z\end{array}\right)$. However, strangely enough, replacing in (3) the column vector $\left(\begin{array}{l}x \\ y \\ z\end{array}\right)$ by the product $\left(\begin{array}{cc}0 & 1 / 5 \\ 1 & 0 \\ 0 & 2 / 5\end{array}\right)\left(\begin{array}{c}5 \\ 10\end{array}\right)$ validates the equality in (3). This explains our remark "not so obviously" in the above Abstract. Indeed

$$
\left(\begin{array}{lll}
0 & 1 & 0 \\
1 & 0 & 2
\end{array}\right)\left(\begin{array}{cc}
0 & 1 / 5 \\
1 & 0 \\
0 & 2 / 5
\end{array}\right)\left(\begin{array}{c}
5 \\
10
\end{array}\right)=\left(\begin{array}{c}
5 \\
10
\end{array}\right)
$$

since $\left(\begin{array}{cc}0 & 1 / 5 \\ 1 & 0 \\ 0 & 2 / 5\end{array}\right)$ is a right inverse of $\left(\begin{array}{lll}0 & 1 & 0 \\ 1 & 0 & 2\end{array}\right)$.

Hence, in view of (4), a solution of (3) is given by:

$$
\left(\begin{array}{l}
x \\
y \\
z
\end{array}\right)=\left(\begin{array}{cc}
0 & 1 / 5 \\
1 & 0 \\
0 & 2 / 5
\end{array}\right)\left(\begin{array}{c}
5 \\
10
\end{array}\right)=\left(\begin{array}{l}
2 \\
5 \\
4
\end{array}\right)
$$

Thus, as (5) shows, a solution of the system (3) is obtained by multiplying on the left the column of the constants by the natural right inverse of the matrix of coefficients of the unknowns.

Clearly, the 2 by 3 matrix of coefficients of the unknowns of the system (3) has rank 2 , and, since there are 3 unknowns involved, the system has infinitely many solutions. The question is what special properties does the solution $x=2, y=5$ and $z=4$ possess. We show below that this particular solution (which is obtained by the use of the natural right inverse) has the smallest Euclidean length, i.e., it is the solution $x=a, y=b, z=c$ of the system ( 3 ) for which $a^{2}+b^{2}+c^{2}$ is minimum.

Theorem. 1. Let $A$ be an $m$ by $n$ matrix of rank $m$ with $m<n$. Then the system of $m$ equations in $n$ unknowns (written in matrix form):

$$
A X=C
$$

has infinitely many solutions and the solution given by

$$
X=A^{\prime}\left(A A^{\prime}\right)^{-1} C
$$

is the unique solution of minimal Euclidean length. 
Proof. That (6) has infinitely many solutions follows from the fact that in (6) the rank of the matrix $A$ is equal to the rank of the augmented matrix $(A \mid C)$ which is equal to $m$ which is less than the number $n$ of the unknowns [1, p.76].

To prove that (7) gives the unique solution of (6) of minimal Euclidean length, let $Y$ be any solution of (6) different from $X$, i.e.,

$$
A Y=A X=0 \text { with } Z=Y-X \neq 0
$$

Thus, $A(Y-X)=A Z=0$ which by (7) implies $X^{\prime} Z=C^{\prime}\left(A A^{\prime}\right)^{-1} A Z=0$. Hence,

$$
X^{\prime} Z=Z^{\prime} X=0
$$

But then, from (8) we have $Y^{\prime} Y=(Z-X)^{\prime}(Z-X)=Z^{\prime} Z-X^{\prime} Z-Z^{\prime} X+X^{\prime} X$ which by (9) implies $Y^{\prime} Y=Z^{\prime} Z+X^{\prime} X$. Recalling that for a column vector $H$ we have $H^{\prime} H=\|H\|^{2}$, the last equality, in view of (8) implies $\|X\|<\|Y\|$. Thus, (6) implies (7), as desired.

As expected, we call a system $S$ of linear equations equivalent to a system $H$ of linear equations if $H$ is obtained from $S$ by the process of linear combinations and elimination of satisfied equations. Moreover, we call a system $S$ of equations consistent if $S$ has a solution. But then, based on Theorem 1 and Remark 1, we have:

Theorem 2. Let $S$ be a consistent system of linear equations which is equivalent to a system $H$ of $k$ linear equations with at least $k$ unknowns. Then $S$ has a unique solution of minimal Euclidean length.

Proof. Clearly, $H$ in its turn is equivalent to a system $M$ of $m$ linear equations with $n$ unknowns with $m \leq n$ whose matrix of coefficients of the unknowns has rank $m$. Now, if $m<n$ then the conclusion' of Theorem 2 follows directly from Theorem 1 . On the other hand, if $m=n$, then by Remark 1 the system $M$ has a unique solution which is also the solution of $M$ of minimal Euclidean length.

Next, we consider the case of systems of linear euqations with more equations than unknowns. The matrix of coefficients of the unknowns of such a system is an $n$ by $m$ matrix with $m<n$, and, in general, such a system has no solution. Thus, this case is not merely the dual of the preceding case where, in general, there is always a solution (in fact, a unique solution of minimal Euclidean length). However, as we shall see below, the natural left inverse of a rectangular matrix is the transpose of the natural right inverse of the transpose matrix.

Let us consider a 3 by 2 matrix $B$, say $\left(\begin{array}{ll}0 & 1 \\ 1 & 0 \\ 0 & 2\end{array}\right)$. It is customary to call a 2 by 3 matrix $L$ a left inverse of $B$ if $L B=I_{2}$. Thus, $\left(\begin{array}{ccc}-2 & 1 & 1 \\ -3 & 0 & 2\end{array}\right)$ as well as $\left(\begin{array}{ccc}2 & 1 & -1 \\ -5 & 0 & 3\end{array}\right)$ 
is a left inverse of $\left(\begin{array}{ll}0 & 1 \\ 1 & 0 \\ 0 & 2\end{array}\right)$. Here also we readily conclude that the above 3 by 2 matrix $B$ has infinitely many left inverses. In fact, let us multiply $B$ on the left by a suitable 2 by 3 matrix $J$ of rank 2 such that the usual inverse $(J B)^{-1}$ of the 2 by 2 square matrix $J B$ exists. Obviously, $(J B)^{-1} J$ is then a left inverse of $B$.

Here again, a natural choice for $J$ is the transpose $B^{\prime}$ of $B$. But then $\left(B^{\prime} B\right)^{-1} B^{\prime}$ is uniquely determined by $B$ and we call it the natural left inverse of $B$. Accordingly,

$$
\begin{aligned}
& \text { the natural left inverse of }\left(\begin{array}{ll}
0 & 1 \\
1 & 0 \\
0 & 2
\end{array}\right) \text { is } \\
& \left(\left(\begin{array}{lll}
0 & 1 & 0 \\
1 & 0 & 2
\end{array}\right)\left(\begin{array}{ll}
0 & 1 \\
1 & 0 \\
0 & 2
\end{array}\right)\right)^{-1}\left(\begin{array}{lll}
0 & 1 & 0 \\
1 & 0 & 2
\end{array}\right)=\left(\begin{array}{ccc}
0 & 1 & 0 \\
1 / 5 & 0 & 2 / 5
\end{array}\right)
\end{aligned}
$$

Remark 2. As in the case of Remark 1 , here also if $B$ is a square $m$ by $m$ matrix of rank $m$ then the natural left inverse of $\left(B^{\prime} B\right)^{-1} B^{\prime}$ of $B$ reduces to the usual inverse $B^{-1}$ of $B$.

Motivated by the above, we introduce:

Definition 2. Let $B$ be an $n$ by $m$ with $m<n$ matrix of rank $m$. Then the $m$ by $n$ matrix $\left(B^{\prime} B\right)^{-1} B^{\prime}$ where $B^{\prime}$ is the transpose of $B$, is called the natural left inverse of $B$.

Now, in contrast to the preceding case, we show that the natural left inverse (and for that matter any left inverse) can be used, in general, to obtain a pseudo solution (and not an actual solution since in general actual solutions may not exist) of $n$ linear equations in $m$ unknowns where $m<n$ and where the rank of the $n$ by $m$ matrix of coefficients of the unknowns is $m$. For instance, let us consider:

$$
\left(\begin{array}{ll}
0 & 1 \\
1 & 0 \\
0 & 2
\end{array}\right)\left(\begin{array}{l}
x \\
y
\end{array}\right)=\left(\begin{array}{l}
5 \\
4 \\
5
\end{array}\right)
$$

Clearly, the system (11) is inconsistent and has no solution. Indeed, it is equivalent to the three equations $x=4, y=5,2 y=5$ and as the last two equations show the system (11) cannot possibly have a solution. However, as (10) shows, the left natural inverse of the matrix of coefficients of the unknowns of the system (11) exists. Thus, there is an obvious attempt (this explains our remark "in an obvious way" in the above Abstract) "to solve" the system (11) by multiplying (on the left) both sides of the equality sign in (11) by $\left(\begin{array}{ccc}0 & 1 & 0 \\ 1 / 5 & 0 & 2 / 5\end{array}\right)$. As a result, we derive:

$$
\left(\begin{array}{ccc}
0 & 1 & 0 \\
1 / 5 & 0 & 2 / 5
\end{array}\right)\left(\begin{array}{ll}
0 & 1 \\
1 & 0 \\
0 & 2
\end{array}\right)\left(\begin{array}{l}
x \\
y
\end{array}\right)=\left(\begin{array}{l}
x \\
y
\end{array}\right)=\left(\begin{array}{ccc}
0 & 1 & 0 \\
1 / 5 & 0 & 2 / 5
\end{array}\right)\left(\begin{array}{l}
5 \\
4 \\
5
\end{array}\right)=\left(\begin{array}{l}
4 \\
3
\end{array}\right)
$$


Clearly, $x=4$ and $y=3$ obtained in (12) is not a solution of the system (11). Indeed.

$$
\left(\begin{array}{ll}
0 & 1 \\
1 & 0 \\
0 & 2
\end{array}\right)\left(\begin{array}{l}
4 \\
3
\end{array}\right)=\left(\begin{array}{l}
3 \\
4 \\
6
\end{array}\right) \neq\left(\begin{array}{l}
5 \\
4 \\
5
\end{array}\right)
$$

However, since $x=4$ and $y=3$ are obtained in a unique way according to our procedure, it may be the case that this assignment of values to $x$ and $y$ has an intrinsic significance in connection with the system (11).

The question is what specific properties does the pseudo solution $x=4$ and $y=3$ possess. To answer the question, we observe that by substituting $x=4$ and $y=3$ in (11), according to (13), we obtain the vector:

$$
\left(\begin{array}{l}
3 \\
4 \\
6
\end{array}\right) \text { instead of }\left(\begin{array}{l}
5 \\
4 \\
5
\end{array}\right) \text { with }\left(\begin{array}{c}
2 \\
0 \\
-1
\end{array}\right) \text { as the error vector }
$$

From (14) it follows that the Euclidean length of the error vector is $\sqrt{(-2)^{2}+(0)^{2}+(1)^{2}}=\sqrt{5}$.

Thus, we see that the assignment of 4 to $x$ and 3 to $y$ in (11) yields an error vector of Euclidean length $\sqrt{5}$, or in short, yields an error of $\sqrt{5}$.

For the sake of simplicity, let us call any assignment of values to the unknowns appearing in a system of equations a pseudo solution of the system. Obviously, an actual solution is also a pseudo solution.

We show below that among all the pseudo solutions of the system (11), the pseudo solution $x=4$ and $y=3$ (which is obtained by the use of the natural left inverse) is the unique pseudo solution of minimal error.

Theorem 3. Let $B$ be an $n$ by $m$ matrix of rank $m$ with $m<n$. Then for the system of $n$ equations in $m$ unknowns (written in matrix form):

$$
B X=C
$$

the pseudo solution given by

$$
X=\left(B^{\prime} B\right)^{-1} B^{\prime} C
$$

is the unique pseudo solution of minimal error (i.e., $C-B X$ has minimal Euclidean length which is also called the best approximate solution).

Proof. To prove that (16) gives the unique pseudo solution of minimal error, we observe that, in view of (16), for any column vector $V$, we have:

$$
(B X-C)^{\prime} B V=\left(B\left(B^{\prime} B\right)^{-1} B^{\prime} C-C\right)^{\prime} B V=C^{\prime}\left(B\left(B^{\prime} B\right)^{-1} B^{\prime}-I\right) B V=0 .
$$


Thus, for every column vector $V$ we have:

$$
(B X-C)^{\prime} B V=V^{\prime} B^{\prime}(B X-C)=0 .
$$

Now, let $Y$ be any vector different from $X$. Thus,

$$
Z=(Y-X) \neq 0 \text { and there fore } B Z \neq 0 \text {. }
$$

But then $(B Y-C)=(B X-C)+B(Y-X)=(B X-C)+B Z$ and therefore $(B Y-$ $C)^{\prime}(B Y-C)=\left((B X-C)^{\prime}+Z^{\prime} B^{\prime}\right)((B X-C)+B Z)$ which, in view of (17) implies $(B Y-C)^{\prime}(B Y-C)=(B X-C)^{\prime}(B X-C)+(B Z)^{\prime}(B Z)$. Consequently, by (18) we have $\|B X-C\|<\|B Y-C\|$. Thus, (15) implies (16), as desired.

Based on Theorem 3 and Remark 2, we have:

Theorem 4. Let $M$ be a system of linear equations with $m$ unknowns and at least $m$ equations whose matrix of coefficients of the unknowns has rank $m$. Then $M$ has a unique pseudo solution of minimal error.

Proof. By the hypothesis of the Theorem, $M$ has $n$ equations with $m \leq n$. Now, if $m<n$ then the conclusion of Theorem 4 follows directly from Theorem 3 . On the other hand, if $m=n$, then by Remark 2 the system $M$ has a unique solution which is also the unique pseudo solution of $M$ of minimal error.

We close by giving two example.

$$
\left(\begin{array}{ccc}
1 & -1 & 2 \\
1 & 2 & 1
\end{array}\right)\left(\begin{array}{l}
x \\
y \\
z
\end{array}\right)=\left(\begin{array}{l}
1 \\
6
\end{array}\right)
$$

The above is a consistent system with more unknowns than equations. It has a solution of minimal Euclidean length which is obtained by multiplying on the left the matrix $\left(\begin{array}{l}1 \\ 6\end{array}\right)$ by the natural right inverse of $\left(\begin{array}{ccc}1 & -1 & 2 \\ 1 & 2 & 1\end{array}\right)$.

The natural right inverse of $\left(\begin{array}{ccc}1 & -1 & 2 \\ 1 & 2 & 1\end{array}\right)$ according to Definition 1, is given as:

$$
\begin{aligned}
\left(\begin{array}{cc}
1 & 1 \\
-1 & 2 \\
2 & 1
\end{array}\right)\left(\left(\begin{array}{ccc}
1 & -1 & 2 \\
1 & 2 & 1
\end{array}\right)\left(\begin{array}{cc}
1 & 1 \\
-1 & 2 \\
2 & 1
\end{array}\right)\right)^{-1} & =\left(\begin{array}{cc}
1 & 1 \\
-1 & 2 \\
2 & 1
\end{array}\right)\left(\begin{array}{ll}
6 & 1 \\
1 & 6
\end{array}\right)^{-1} \\
& =\frac{1}{35}\left(\begin{array}{cc}
5 & 5 \\
-8 & 13 \\
1.1 & 4
\end{array}\right)
\end{aligned}
$$

and therefore, the unique solution of minimal Euclidean length of (19) is given by:

$$
\left(\begin{array}{l}
x \\
y \\
z
\end{array}\right)=\frac{1}{35}\left(\begin{array}{cc}
5 & 5 \\
-8 & 13 \\
11 & 4
\end{array}\right)\left(\begin{array}{l}
1 \\
6
\end{array}\right)=\left(\begin{array}{l}
1 \\
2 \\
1
\end{array}\right) \text {, i.e., } x=1, y=2, z=1
$$


Finally, let us consider:

$$
\left(\begin{array}{cc}
1 & 1 \\
-1 & 2 \\
2 & 1
\end{array}\right)\left(\begin{array}{l}
x \\
y
\end{array}\right)=\left(\begin{array}{l}
3 \\
1 \\
2
\end{array}\right)
$$

The above is a system with 3 equations and 2 unknowns whose matrix of coefficients of the unknowns has rank 2. It has no solution. However, by Theorem 4, it has a unique pseudo solution of minimal error which is obtained by multiplying on the left the matrix $\left(\begin{array}{l}3 \\ 1 \\ 2\end{array}\right)$ by the natural left inverse of $\left(\begin{array}{cc}1 & 1 \\ -1 & 2 \\ 2 & 1\end{array}\right)$

The natural left inverse of $\left(\begin{array}{cc}1 & 1 \\ -1 & 2 \\ 2 & 1\end{array}\right)$ according to Definition 2, is given as:

$$
\begin{aligned}
\left(\left(\begin{array}{ccc}
1 & -1 & 2 \\
1 & 2 & 1
\end{array}\right)\left(\begin{array}{cc}
1 & 1 \\
-1 & 2 \\
2 & 1
\end{array}\right)\right)^{-1}\left(\begin{array}{ccc}
1 & -1 & 2 \\
1 & 2 & 2
\end{array}\right) & =\left(\begin{array}{cc}
6 & 1 \\
1 & 6
\end{array}\right)^{-1}\left(\begin{array}{ccc}
1 & -1 & 2 \\
1 & 2 & 1
\end{array}\right) \\
& =\frac{1}{35}\left(\begin{array}{ccc}
5 & -8 & 11 \\
5 & 13 & 4
\end{array}\right)
\end{aligned}
$$

and therefore, the unique pseudo solution of minimal error of (20) is given by :

$$
\begin{gathered}
\left(\begin{array}{l}
x \\
y
\end{array}\right)=\frac{1}{35}\left(\begin{array}{ccc}
5 & -8 & 11 \\
5 & 13 & 4
\end{array}\right)\left(\begin{array}{l}
3 \\
1 \\
2
\end{array}\right)=\left(\begin{array}{l}
29 / 35 \\
36 / 35
\end{array}\right), \\
\text { i.e., } x=29 / 35, y=36 / 35
\end{gathered}
$$

This paper is a considerable amplification (with the collaboration of the second author) of the first author's abstract [2].

\section{References}

[1] Bronson, R. "Matrix Methods". Academic Press, New York, 1970.

[2] Abian, A. "The Natural Right and the Natural Left Inverses of Rectangular Matrices". Amer. Math. Soc. Abstracts 5(1984) 394.

Department of Mathematics, Iowa State University, Ames, Iowa 50011, U.S.A.

Department of Mathematics, Southwest Missouri State University, Springfield, Missouri 65803, U.S.A. 